\title{
Functional Aspects of a Second Mechanism of Candidacidal Activity by Human Neutrophils
}

\author{
ROBERT I. LEHRER \\ From the Cancer Research Institute and the Department of Medicine, School of \\ Medicine, University of California, San Francisco, California 94122
}

A в S TRACT We tested the ability of human neutrophils to kill five Candida species and the yeast Torulopsis glabrata. C. parapsilosis and C. pseudotropicalis were found to be killed readily by normal and myeloperoxidase-deficient neutrophils and were selected to probe the myeloperoxidase-independent fungicidal mechanisms of the neutrophil.

These organisms were killed with relatively normal (C. parapsilosis) or moderately reduced (C. pseudotropicalis) effectiveness by neutrophils from two boys with X-linked chronic granulomatous disease. Azide (2 $\mathrm{mm}$ ) and sulfadiazine $(4 \mathrm{~mm})$ exerted a relatively small inhibitory effect on the ability of normal neutrophils to kill $C$. parapsilosis. These compounds did not, however, inhibit the killing of $C$. parapsilosis by myeloperoxidase-deficient neutrophils, although they blocked their iodination of ingested Candida cells. Anaerobic incubation conditions inhibited the ability of normal neutrophils to kill $C$. parapsilosis slightly but did not impair this function in myeloperoxidase-deficient cells.

All of the Candida species tested had catalase activity, yet their sensitivity to $\mathrm{H}_{2} \mathrm{O}_{2}$ in cell-free systems varied considerably. Our $C$. parapsilosis strain was extraordinarily resistant to $\mathrm{H}_{2} \mathrm{O}_{2} \quad\left(\mathrm{LD}_{75}: 0.14 \mathrm{M}\right)$, as compared with $C$. pseudotropicalis or with our reference strain of $C$. albicans ( $\mathrm{LD}_{75}: 2.3 \times 10^{-3} \mathrm{M}$ and $3.4 \times 10^{-3}$ I, respectively).

These data establish the existence in human neutrophils of a second mechanism that exerts microbicidal activity against certain Candida species; the mechanism is unrelated to myeloperoxidase, iodination, or to the direct effects of $\mathrm{H}_{2} \mathrm{O}_{2}$ generated by the endogenous metabolic processes of the neutrophil. As yet unidentified, this mechanism appears to remain operative in

An abstract was presented to the national meeting of the American Federation for Clinical Research, Atlantic City, N. J., April 1972.

Received for publication 22 February 1972 and in revised form 14 June 1972. the neutrophils of subjects with hereditary myeloperoxidase deficiency or chronic granulomatous disease.

\section{INTRODUCTION}

Despite progress in our understanding of the phagocytic process, the actual mechanisms whereby human neutrophils kill microorganisms are incompletely understood. Myeloperoxidase (MPO) ${ }^{1}$ participates in some of these bactericidal and fungicidal processes, and the role of this enzyme in the microbicidal activity of intact normal neutrophils may be considerable for certain organisms (1-3).

MPO-deficient individuals may remain in good health. without excessive or unusual infections, for prolonged periods of time; this fact is difficult to reconcile with the presumed importance of MPO to the economy of the neutrophil. Recently, Klebanoff and Pincus have attempted to explain this paradox by suggesting that peroxidase-deficient neutrophils can exert microbicidal activity by a compensatory increase in the nonenzymatic effects of endogenously generated $\mathrm{H}_{2} \mathrm{O}_{2}$ (4).

We have previously shown that human neutrophils with deficiencies in MPO have an impaired ability to kill certain bacteria and the fungus, Candida albicans $(2,3)$. We now report the ability of human neutrophils to kill several other species of yeast-like fungi and present evidence for the existence of a fungicidal mechanism that is independent of MPO and that is not a consequence of the direct action of $\mathrm{H}_{2} \mathrm{O}_{2}$. Such mechanisms offer an alternative explanation for the microbicidal activity of MPO-deficient neutrophils.

\section{METHODS}

Fungi. The organisms used in these studies included three strains of $C$. albicans: strain 820 (5), strain B-311, and strain Mo (a recent isolate from a patient with systemic

${ }^{1}$ Abbreviations used in this paper: CFU, colony-forming unit: MPO, myeloperoxidase. 
candidiasis); three strains of $C$. tropicalis, including one recently recovered from a patient with Candida pyelonephritis; two strains each of $C$. parapsilosis and $C$. krusei; one strain of $C$. pseudotropicalis; and four strains of Torulopsis glabrata. ${ }^{2}$

The fungi were grown in Sabouraud's $2 \%$ dextrose broth ${ }^{3}$ except for $C$. pseudotropicalis, which was cultured exclusively in tryptose phosphate broth, ${ }^{4}$ and $C$. parapsilosis, which was grown either in Sabouraud's or tryptose phosphate broth. The cultures were incubated at $33^{\circ} \mathrm{C}$ for $2-3$ days ( $C$. pseudotropicalis and $C$. parapsilosis), 5-7 days ( $C$. albicans), or 3-7 days (all others). Under these growth conditions, the fungi manifested the following characteristics: (a) stationary growth phase with high viability; (b) few autolysed or mycelial-phase cells; ${ }^{5}$ (c) good agreement between observed colony counts and those predicted by enumeration of colony-forming units (CFU) with an hemocytometer; (d) a lag phase of at least $90 \mathrm{~min}$ before the colony count fell ( $C$. albicans) or rose significantly (all others) after the introduction of fungi to medium; and $(e)$ a mean of $1.3-2.0$ cells per CFU.

Fungi were washed twice with sterile distilled water containing $0.01 \%$ gelatin (gel-water), counted in an hemocytometer, and suspended at the desired concentration in gel-water or phosphate-buffered saline.

Leukocytes. Mixed peripheral blood leukocytes (containing $55-75 \%$ neutrophils and $2-7 \%$ monocytes) were prepared as previously described (5). Purified neutrophil preparations (85-95\% neutrophils, less than $0.2 \%$ monocytes) were prepared by a minor modification of Böyum's technique (6) ; a mixture of 24 vol of $9 \%$ Ficoll $^{\circ}$ and 10 vol of $32.6 \%$ sodium diatrizoate ${ }^{7}$ replaced his Isopaque-Ficoll formulation.

Leukocytes were obtained from normal subjects, a subject (C. J. B.) with hereditary myeloperoxidase deficiency (2), and two boys (M. B. and D. D.), aged 8 and $9 \mathrm{yr}$, who have classic X-linked chronic granulomatous disease. At the time of these studies, C. J. B. continued to be free of all significant infections, M. B. had evidence of resolving, suppurative cervical adenitis (Neisseria sp.), and D. D. had recovered some 2 months earlier from severe bilateral pneumonitis, thought to have been caused by Aspergillus fumigatus.

Acrobic assay's. Aerobic assays were conducted in $12 \times 75$ $\mathrm{mm}$ polystyrene tubes ${ }^{8}$ that contained, in a final volume of

${ }^{2} \mathrm{I}$ am indebted to Dr. C. Halde for providing and identifying many of the Candida isolates, to Dr. H. C. Hasenclever for providing C. albicans B-311, to Dr. S. J. Klebanoff for providing a strain of $C$. tropicalis used in his earlier experiments, and to Dr. T. Eickhoff for providing three strains of $T$. glabrata.

${ }^{3}$ Difco Laboratories, Detroit, Mich., or BBL, Division of BioQuest, Cockeysville, Md.

'Difco Laboratories, Detroit, Mich.

${ }^{5}$ Certain lots of Sabouraud's broth available from commercial suppliers proved unsatisfactory for these tests. In them, fungi attained a relatively low final concentration in stationary phase, and numerous $(20-30 \%)$ nonviable and autolysed cells were present. Such preparations would have provided unacceptably high background values for the specific staining assay; therefore, lots of broth were screened for their ability to produce maximally viable fungal cultures before they were used in these studies.

- Pharmacia, Uppsala, Sweden.

${ }^{7}$ Hypaque, Winthrop Laboratories, New York.

${ }^{8}$ Falcon Plastics, Division of BioQuest, Los Angeles, Calif.
$1.6 \mathrm{ml}, 0.4 \mathrm{ml}$ of normal group AB serum, leukocytes sufficient to provide $4 \times 10^{\circ}$ neutrophils, Hanks' balanced salt solution $^{\circ}$ and fungi. After the other components had been incubated together for $15 \mathrm{~min}$ at $37^{\circ} \mathrm{C}$, fungi were added at ratios that approximated one CFU per two neutrophils in studies with mixed leukocyte populations and one CFU per five neutrophils in studies with purified neutrophil preparations. After addition of fungi, the tubes were thoroughly mixed and triplicate samples withdrawn for dilution, plating, and colony counting. The tubes were then incubated at $37^{\circ} \mathrm{C}$ while rotating end-over-end at $30 \mathrm{rpm}$. Additional triplicate samples were secured for colony counting after $45 \mathrm{~min}$ (in some experiments), $90 \mathrm{~min}$, and $180 \mathrm{~min}$ of incubation. In experiments wherein the colony-count results were to be compared with the specific-staining method of evaluating candidacidal activity, samples were also removed after 150 min of incubation and slides were prepared, stained, and interpreted as previously described (7). Sabouraud's 2\% dextrose agar ${ }^{10}$ was used in the colony-count experiments; the plates were incubated for $48-96 \mathrm{hr}$ at $33^{\circ} \mathrm{C}$ to permit maximal colony development before being counted.

Anaerobic assay's. Anaerobic assays were conducted in $15-\mathrm{ml}$ polycarbonate plastic tubes with tightly fitting sleevetype rubber stoppers. The final volume in these tubes was $4.0 \mathrm{ml}$ and contained leukocytes, fungi, and serum in the same relative proportions as were present in the aerobic experiments with purified neutrophils. The tubes were capped, and those to be rendered anaerobic were flushed for 30 min with a stream of highly purified nitrogen ${ }^{11}$ that was passed through an alkaline solution of pyrogallol (8) and then through distilled water before entering the tubes via appropriately sized and placed needles. The fungi to be added were similarly capped, flushed with nitrogen, and inoculated into the assay tubes through the stopper with appropriate precautions to exclude contamination by room air. At intervals $(45,90$, and $180 \mathrm{~min}$ ) samples were withdrawn from the incubation mixtures by syringe, and these were diluted and prepared for colony counting as described above. Slides were prepared from the samples taken at $45 \mathrm{~min}$ and examined to evaluate the extent of phagocytosis.

At the conclusion of the experiment, the residual contents of representative tubes were found to have an oxygen tension less than $1 \%$ that of air-saturated medium similarly tested in a Gilson Model KM oxygraph with a Clark electrode. ${ }^{12}$ To further confirm the adequacy of this technique of achieving anaerobiosis, leukocytes treated in an analogous manner were shown to have no stimulation of hexosemonophosphate shunt activity after ingestion of heat-killed Candida cells.

Quantitatiz'e iodination. The ability of normal or MPOdeficient neutrophils to iodinate ingested microorganisms was tested by a modification of the method of Pincus and Klebanoff (9). The standard reaction mixture consisted of $2.5 \times 10^{8}$ neutrophils (prepared by the Hypaque-Ficoll method described above), $10 \%$ normal group AB serum, 10 nmoles of $\mathrm{NaI}\left(0.2 \mu \mathrm{Ci}\right.$ of $\left.{ }^{125} \mathrm{I}\right)$, approximately 700 nmoles of glucose (including that added in the serum component), and calcium-free Krebs-Ringer phosphate buffer, in a final volume of $0.5 \mathrm{ml}$. To this were added the particles: $5 \times 10^{\circ}$

\footnotetext{
${ }^{\ominus}$ Grand Island Biological Co., Berkeley, Calif.

${ }^{10}$ Difco Laboratories, Detroit, Mich.

${ }^{11} \mathrm{Hi}$-pure grade, Liquid Carbonic Division, General Dynamics Corp., New York.

${ }^{12}$ Yellow Springs Instrument Co., Inc., Yellow Springs,
} Ohio. 
heat-killed $C$. albicans, or, in some experments, $3 \times 10^{\circ}$ viable $C$. parapsilosis. Tubes were incubated for $60 \mathrm{~min}$ and processed as described (9).

Effect of hydrogen peroxide. The susceptibility of various Candida species to hydrogen peroxide was measured by minor modifications of a previously described technique (10). Fungi were washed twice with, and then suspended in, distilled water. They were exposed to various concentrations of $\mathrm{H}_{2} \mathrm{O}_{2}$ in tubes containing, in a final volume of 0.5 $\mathrm{ml}$, approximately $1 \times 10^{6} \mathrm{CFU}$ of fungi, citrate-phosphate buffer ( $50 \mu$ moles $)$, and water. The reactions were started by adding $\mathrm{H}_{2} \mathrm{O}_{2}$ (or water), and the tubes were incubated for $45-60 \mathrm{~min}$ at $37^{\circ} \mathrm{C}$. Colony counts were then obtained by the usual method. During the incubations with $\mathrm{H}_{2} \mathrm{O}_{2}$, the evolution of bubbles was noted with all species tested, indicating that all contained catalase.

\section{RESULTS}

\section{Measurements of fungicidal activity}

The first series of experiments was performed with mixed peripheral blood leukocytes. Although such preparations also contained monocytes and eosinophils, well over $90 \%$ of the added organisms were ingested by neutrophils. Accordingly, these studies were taken to indicate neutrophil function. The assay conditions insured that the added fungi were rapidly ingested, and few yeasts remained in extracellular locations after 15 min of incubation.

The susceptibility of various Candida species to normal and MPO-deficient neutrophils is shown in Figs.

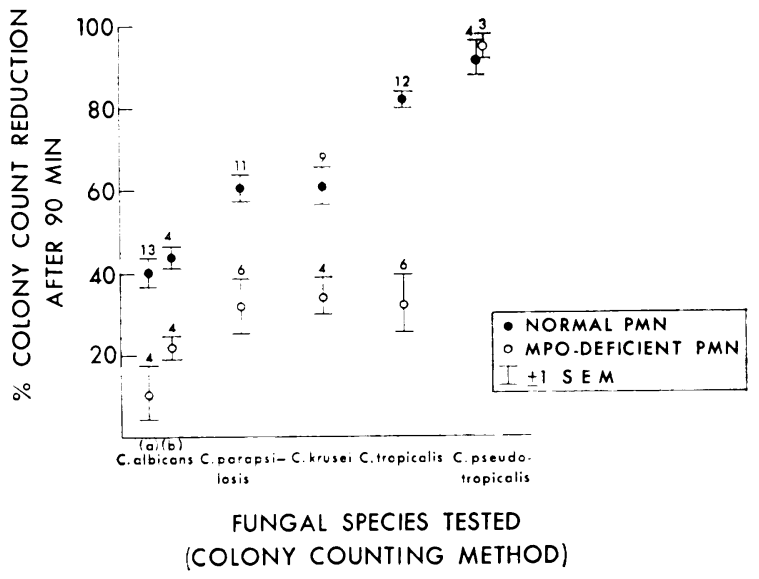

Figure 1 Ability of normal and MPO-deficient neutrophils to kill Candida species. Results with $C$. albicans strain 820 are indicated by $(a) ; C$. albicans strains $\mathrm{Mo}$ and $\mathrm{B}-311$ have been combined $(b)$. Results with two strains each of $C$. parapsilosis and $C$. krusei and with three strains of $C$. tropicalis were concordant within each species, and have been combined. The ordinate shows the mean percentage reduction (from original colony count levels) $90 \mathrm{~min}$ after fungi were added to mixed peripheral blood leukocytes. The numbers of experiments with different normal subjects and of experiments (each on a different day) with C. J. B.'s MPO-deficient leukocytes are shown above the bars denoting 1 SEM.
1 and 2 . In both figures, killing by neutrophils is reflected by the percentage fall from initial colony counts. Fig. 2 shows, in addition, the percentage of Candida cells within neutrophils that have acquired the Giemsa-staining characteristics of ghosts after an incubation period of $2.5 \mathrm{hr}$.

The results with both methods, colony counting and specific staining, are reasonably concordant. Under these test conditions, the various Candida species differed in their sensitivity to normal neutrophils, ranging from the somewhat resistant $C$. albicans to the highly susceptible $C$. pseudotropicalis. The Candida species showed an even greater range of susceptibility to MPOdeficient neutrophils; the ability of these leukocytes to kill $C$. albicans was decidedly impaired whereas their ability to kill $C$. parapsilosis or $C$. pseudotropicalis was relatively or completely intact.

In another experiment, the ability of normal and MPO-deficient neutrophils to kill four strains of $T$. glabrata was compared (Fig. 3). Although killing of this organism by the peroxidase-deficient cells proceeded at a subnormal rate, it reached approximately normal levels at the end of $3 \mathrm{hr}$ of incubation. One of these $T$. glabrata isolates (strain D) was also studied in an additional series of experiments with normal and MPOdeficient mixed leukocytes, and this same pattern of delayed, but ultimately normal, killing by the MPOdeficient cells was seen consistently.

\section{Characterization of the candidacidal mechanism}

In the experiments summarized in Figs. 1 and 2, mean percentage reductions in colony counts ( \pm SEM) with $C$. parapsilosis, strain $12-10$, were $42 \%( \pm 4.4 \%)$ for MPO-deficient leukocytes and $61.8 \%( \pm 4.9 \%)$ for normal leukocytes after $90 \mathrm{~min}$; after $180 \mathrm{~min}$, MPOdeficient leukocytes had a $72 \%( \pm 3.8 \%)$ reduction and normal leukocytes had a $79 \%( \pm 4.2 \%)$ reduction. C. parapsilosis, strain $12-10$ and $C$. pseudotropicalis appeared to be suitable species for exploring the nature of the MPO-independent candidacidal mechanisms of the human neutrophil. Accordingly, experiments were conducted with these organisms to elucidate the following points: $(a)$ the effects of azide and sulfadiazine on the candidacidal function and the iodinating capacity of normal and MPO-deficient neutrophils; $(b)$ the candidacidal activity of neutrophils from children with chronic granulomatous disease; $(c)$ the effects of anaerobiosis on neutrophil candidacidal activity: and (d) the comparative sensitivity of $C$. parapsilosis, $C$. pseudotropicalis, and $C$. albicans to the direct toxic effects of $\mathrm{H}_{2} \mathrm{O}_{2}$.

Effects of azide and sulfadiazine. Azide and sulfadiazine are known to inhibit the function of MPO-mediated bactericidal and candidacidal activity (C. albicans) 


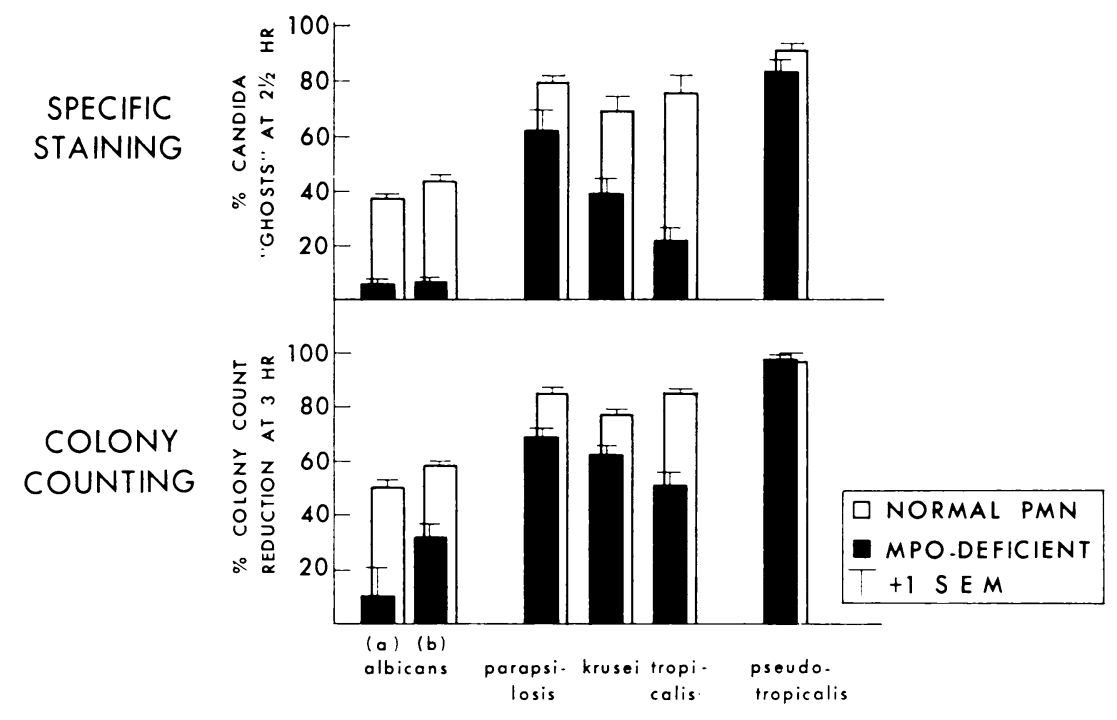

CANDIDA SPECIES TESTED

FIGURE 2 Ability of normal and MPO-deficient neutrophils to kill Candida species. Top. percentage of Candida cells within neutrophils that were killed and digested after $2.5 \mathrm{hr}$ of incubation, as determined by the specific staining procedure: bottom, percentage fall in colony count $3 \mathrm{hr}$ after addition of fungi to mixed peripheral blood leukocytes. Other experimental conditions are as described in Fig. 1.

in the normal human neutrophil (1, 11). The effect of these two agents on the ability of normal and MPOdeficient neutrophils to kill $C$. parapsilosis is shown in Fig. 4. Both sulfadiazine (t mm) and azide (2 mM) caused a consistent. but relatively small, decrease in the ability of normal neutrophils to kill this organism after $90 \mathrm{~min}$ (not shown) and $180 \mathrm{~min}$. Sulfadiazine was slightly more effective than was azide in this regard. A combination of sulfadiazine and azide had inhibitory effects that were about as effective as azide alone: no potentiation of inhibition was observed. In contrast, azicle. sulfarliazine. or a combination of both caused a slight enhancement of the candidacidal activity of MIPO-deficient neutrophils. This effect was
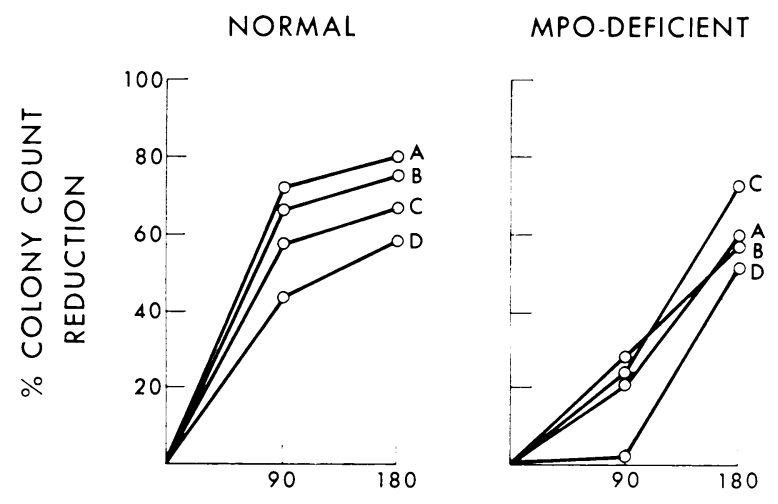

INCUBATION TIME (minutes)

FIGLRE 3 Ability of normal and MPO-deficient neutrophils to kill Torulopsis glabrata. Four strains of $T$. glabrata (A, B, C, D) were added to mixed peripheral blood leukocytes as described in the text. Colony counts in cell-free control tubes averaged 106.7 and $118.7 \%$ of initial values at 90 and 180 min, respectively.

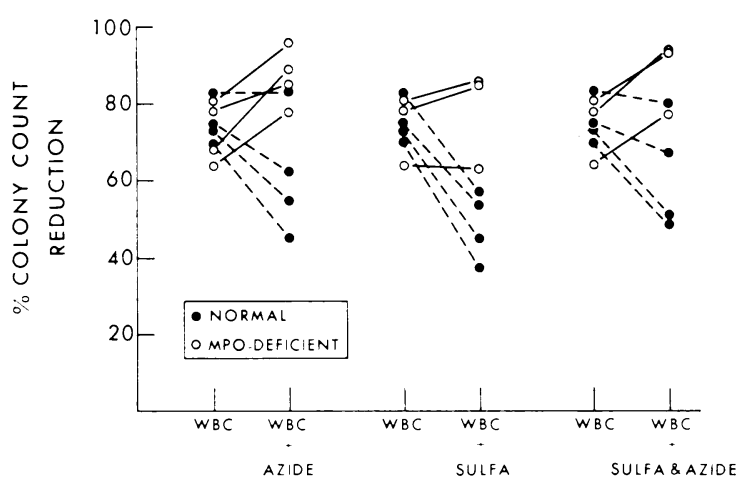

Figr 4 Elfect of azicle $(2 \mathrm{mMr})$ and sulfadiazine $(4 \mathrm{mM})$ on the ability of normal and MPO-deficient leukocytes to kill (. parapilosis, strain 12-10. Assays were performed with mixed peripheral blocd leukocytes incubated with fungi for $3 \mathrm{hr}$. Aiter $3 \mathrm{hr}$, colony counts in cell-free controls were (mean $\pm s E M) ~ 118.3 \pm 22.5 \%$ (balanced salt solution), 92.0 $\pm 5.6 \%$ (sodium azide), $98.0 \pm 7.4 \%$ (sulfadiazine), 94.8 $\pm 14.3 \%_{c}$ (sulfadiazine + azide) of the initial values. 
somewhat more prominent after $90 \mathrm{~min}$ of incubation (not shown).

Quantitative iodination. Iodination of ingested microorganisms has been proposed as a microbicidal mechanism in the human neutrophil (12). When we measured the ability of MPO-deficient neutrophils to iodinate ingested $C$. albicans cells, we were surprised to observe that a substantial degree of iodination occurred, attaining a mean of $41.2 \%$ of normal activity in five separate paired experiments. Three of these experiments, summarized in Table I, show that viable C. parapsilosis cells were iodinated by normal and (less well) by MPO-deficient neutrophils. Azide, sulfadiazine, and aminotriazole caused essentially complete inhibition of the iodinating capacity of MPO-deficient leukocytes.

Chronic granulomatous disease. Neutrophils from children with $\mathrm{X}$-linked chronic granulomatous disease of childhood do not kill ingested $C$. albicans in vitro $(5,7)$. In contrast, their ability to deal with $C$. parapsilosis and C. pseudotropicalis is shown in Fig. 5. It is apparent that the neutrophils of the two boys had an appreciable ability to kill $C$. pseudotropicalis and could kill $C$. parapsilosis with normal or nearly normal efficacy. As such affected neutrophils almost certainly develop subnormal intracellular concentrations of $\mathrm{H}_{2} \mathrm{O}_{2}$, these results suggested that human neutrophils might contain a mechanism other than MPO or endogenously produced $\mathrm{H}_{2} \mathrm{O}_{2}$ that could kill these Candida species.

The results with MPO-deficient neutrophils in Fig. 5 show a higher absolute ability to kill $C$. parapsilosis

TABLE I

Iodination of Candida by Normal and MyeloperoxidaseDeficient Neutrophils

\begin{tabular}{llcc}
\hline & & \multicolumn{2}{c}{ Iodination* by } \\
\cline { 2 - 3 } Particlet & \multicolumn{1}{c}{ Agent } & $\begin{array}{c}\text { MPO- } \\
\text { deficient } \\
\text { neutrophils }\end{array}$ & $\begin{array}{c}\text { Normal } \\
\text { neutrophils }\end{array}$ \\
\hline None & None & 0.92 & 0.85 \\
C. albicans & None & 2.02 & 4.06 \\
& Azide & 0.10 & 0.20 \\
& Sulfadiazine & 0.13 & 0.57 \\
C. parapsilosis & Aminotriazole & 0.08 & 0.26 \\
& None & 1.21 & 2.23 \\
& Azide & 0.06 & 0.18 \\
& Sulfadiazine & 0.13 & 0.47 \\
& Aminotriazole & 0.07 & 0.22 \\
\hline
\end{tabular}

* Iodination expressed as nmoles of iodide converted to acid-precipitable form per $1 \times 10^{7}$ neutrophils in $1 \mathrm{hr}$. Each result is the mean of three paired experiments with a different normal subject and with C. J. B. Within a given experiment, each value is the mean of triplicate samples. (Less than 0.10 nmoles of iodide was "fixed" to C. albicans or C. parapsilosis in cellfree controls.)

$\ddagger$ All tubes contained neutrophils and other components as described in the text.

$\$$ Added at the following concentrations: azide and aminotriazole, $2 \mathrm{~mm}$; sulfadiazine, $4 \mathrm{~mm}$.

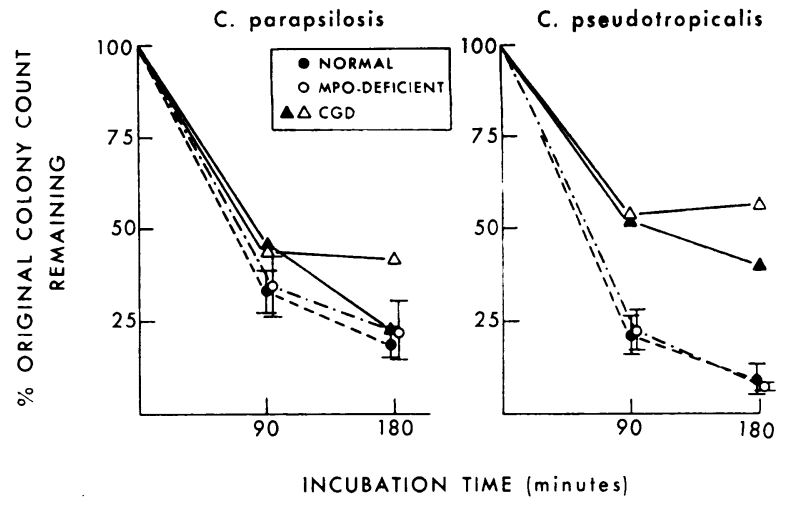

FIGURE 5 Ability of normal, MPO-deficient, and chronic granulomatous disease neutrophils to kill Candida species. The ordinate shows the percentage of originally added colonies that remained viable after incubation for specified periods with purified neutrophil populations from seven normal subjects (๑), MPO-deficient neutrophils from C. J. B. (four experiments $\bigcirc$ ), and neutrophils from M. B. and D. D., two children with chronic granulomatous disease (CGD) $(\triangle, \boldsymbol{\Delta})$. In two of these experiments, candidacidal activity was also measured at $45 \mathrm{~min}$ with normal and MPO-deficient neutrophils. The mean percentage of $C$. parapsilosis remaining viable at 45,90 , and $180 \mathrm{~min}$ in these two experiments was $34.6,21.9$, and $15.4 \%$ (normal) and $29.8,26.2$, and $13 \%$ (MPO-deficient). In the experiments with M. B. and D. D., 48.5 and $35.4 \%$ of $C$. parapsilosis remained viable after $45 \mathrm{~min}$. Cell-free control tubes contained (mean \pm SEM) $105.4 \pm 5.8 \%$ (C. pseudotropicalis) and $99.2 \pm 5.3 \%$ (C. parapsilosis) of the original colony counts after $90 \mathrm{~min}$ of incubation, and $144.8 \pm 14.9 \%(C$. pseudotropicalis) and $120.2 \pm 7.7 \%$ (C. parapsilosis) after $3 \mathrm{hr}$.

after 90 min than is shown in Fig. 1. Although technical considerations may suffice to explain these differences, we cannot exclude alterations in characteristics of the organism or in performance of the MPO-deficient leukocytes during the interval separating the two sets of experiments. Technical differences can be summarized as follows: experiments summarized in Fig. 5 were done $6-8$ months later; purified neutrophil populations were used in place of mixed leukocytes; and only one strain of $C$. parapsilosis, strain 12-10, was used after incubation for 2 or 3 days in tryptose phosphate broth, rather than combining the results with two strains that had been incubated for 5 days in Sabouraud's dextrose broth.

Anaerobic conditions. We compared the effects of anaerobic conditions on the ability of normal and MPOdeficient neutrophils to kill $C$. parapsilosis. We reasoned that if either normal or peroxidase-deficient leukocytes were exerting candidacidal activity by virtue of the system operative in the leukocytes of children with chronic granulomatous disease, they might continue to function well under anaerobic conditions. On the other hand, if candidacidal activity depended on the capacity 
of either cell type to produce normal or elevated concentrations of $\mathrm{H}_{2} \mathrm{O}_{2}$, anaerobic conditions should greatly inhibit killing. Anaerobiosis caused a relatively slight decrease in the function of normal leukocytes, similar to that observed in the earlier experiments with azide, but it did not inhibit the function of MPO-deficient leukocytes (Fig. 6).

Sensitivity of the Candida species to hydrogen peroxide. The likelihood that normal or MPO-deficient neutrophils employed $\mathrm{H}_{2} \mathrm{O}_{2}$ itself to kill these organisms was examined indirectly by studies wherein we determined the susceptibilities of $C$. parapsilosis, $C$. pseudotropicalis, and the reference strain of $C$. albicans (UC 820) to $\mathrm{H}_{2} \mathrm{O}_{2}$ (Fig. 7).

\section{DISCUSSION}

Previous studies from this laboratory have indicated that the ability of human neutrophils to kill $C$. albicans derives from the operation of a microbicidal system that includes myeloperoxidase and hydrogen peroxide $(2,5$, 10). This system, originally described by Klebanoff, is inhibited by azide (1) and sulfadiazine (11), and its operation in intact neutrophils is attended by iodination of the ingested microorganisms (1). It has been suggested that iodination may serve as an actual intraleukocytic microbicidal event mediated by this system (12), but this hypothesis has been seriously challenged (13).

Although Pincus and Klebanoff reported that MPOdeficient leukocytes iodinated ingested Lactobacillus acidophilus only about $7.6 \%$ (heat-killed organisms) or $16.8 \%$ (viable organisms) as effectively as normal leukocytes (9), we found a much smaller abnormality in their ability to iodinate heat-killed $C$. albicans. This iodination, which reached approximately one-half of normal levels, was blocked by aminotriazole, suggesting that it may have been mediated by catalase. Klebanoff has

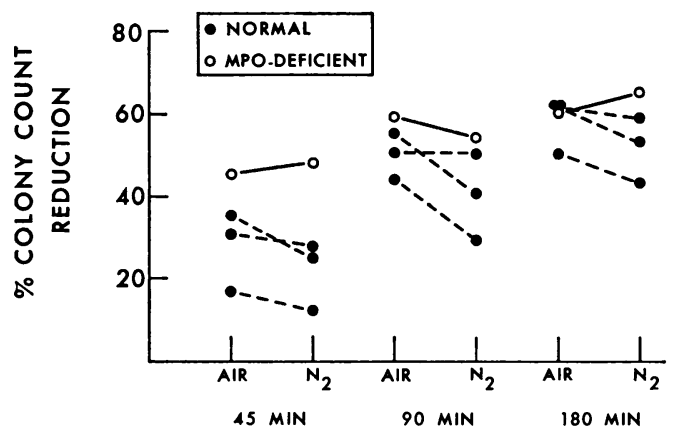

INCUBATION TIME AND CONDITIONS

FIGURE 6 Effect of anaerobic conditions on ability of normal and MPO-deficient neutrophils to kill $C$. parapsilosis 12-10. Purified neutrophil populations of three normal subjects (๑) and of the MPO-deficient subject C. J. B. (O) were used.

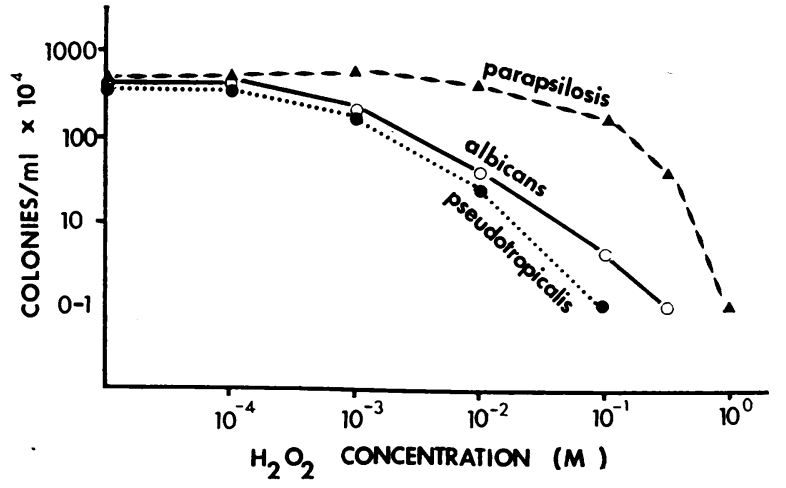

Figure 7 Relative susceptibility of Candida species to hydrogen peroxide. Absolute values of colony counts after exposure of the Candida species to $\mathrm{H}_{2} \mathrm{O}_{2}$ at $\mathrm{pH} 5.0$ are shown under conditions described in the text. Similar results were obtained at $\mathrm{pH} 6.0$ and 7.0. $\Delta, C$. parapsilosis; $\mathrm{O}$, C. albicans; •, C. psendotropicalis.

shown that mixtures of catalase, $\mathrm{H}_{2} \mathrm{O}_{2}$, and iodide can catalyze the iodination of microorganisms in vitro and can exert microbicidal activity (14). However, the observation that azide and sulfadiazine blocked the ability of MPO-deficient neutrophils to iodinate ingested Candida species, but did not interfere with their ability to kill $C$. parapsilosis, indicates that iodination is not the mechanism of this candidacidal activity.

Was the ability of normal or MPO-deficient neutrophils to kill $C$. parapsilosis a consequence of the direct effects of endogenously generated $\mathrm{H}_{2} \mathrm{O}_{2}$ ? This possibility is suggested by recent studies of Klebanoff and Pincus, who reported that the leukocytes of our MPO-deficient patient (C. J. B.) developed a greater than normal increase in activity of the hexose-monophosphate shunt, and in oxidation of formate, after exposure to polystyrene microspheres (4). Although such studies indicate alterations in the activity of pathways that use $\mathrm{H}_{2} \mathrm{O}_{2}$, they do not necessarily indicate changes in the rate of generation or accumulation of $\mathrm{H}_{2} \mathrm{O}_{2}$ within MPO-deficient neutrophils; however, we evaluated the possible contribution of $\mathrm{H}_{2} \mathrm{O}_{2}$ to the ability of MPO-deficient neutrophils to kill certain Candida species.

Neutrophils from children with X-linked chronic granulomatous disease have a genetically determined abnormal metabolic response to phagocytosis that includes a markedly reduced ability to generate $\mathrm{H}_{2} \mathrm{O}_{2}(15)$. That such neutrophils manifest a normal or relatively intact ability to kill $C$. parapsilosis and $C$. psendotropicalis indicates that these species are susceptible to some microbicidal system that does not depend on the production of supranormal (or even normal) concentrations of $\mathrm{H}_{2} \mathrm{O}_{2}$. Anaerobic incubation did not decrease the candidacidal activity of normal neutrophils substantially, suggesting that the same system operates in normal neutrophils. If 
MPO-deficient neutrophils compensated for their primary abnormality by a secondary increase in the direct effects of $\mathrm{H}_{2} \mathrm{O}_{2}$, their ability to kill $C$. parapsilosis should have been even more sensitive to anaerobiosis than that of normal neutrophils. In fact, these MPO-deficient neutrophils were less sensitive, and the effect of anaerobic conditions resembled that of azide. Finally, if the hypothesis advanced by Pincus and Klebanoff is correct and serves as the basis of MPO-independent candidacidal activity in the human neutrophil, then the ability of MPOdeficient leukocytes to kill Candida species should be in direct proportion to the susceptibility of these fungi to $\mathrm{H}_{2} \mathrm{O}_{2}$. However, MPO-deficient neutrophils killed $C$. parapsilosis normally, but had little effect on a strain of C. albicans that was 50 -fold more sensitive to $\mathrm{H}_{2} \mathrm{O}_{2}$ in a cell-free system.

We conclude, therefore, that a distinct microbicidal system in the human neutrophil exists that is independent of MPO, iodination, or the direct effects of endogenously generated $\mathrm{H}_{2} \mathrm{O}_{2}$. Narrowly interpreted, these data can provide an explanation for the ability of MPO-deficient neutrophils and those from patients with chronic granulomatous disease to kill certain species of Candida. More broadly interpreted, these studies should serve to redirect our attention to the importance of microbicidal systems in the human neutrophil that are not primarily dependent on the remarkable oxidative metabolic events that occur after phagocytosis.

\section{ACKNOWLEDGMENTS}

This study was supported by grants CA-11067 and AI-10547 from the U. S. Public Health Service.

\section{REFERENCES}

1. Klebanoff, S. J. 1970. Myeloperoxidase: contribution to the microbicidal activity of intact leukocytes. Science (Wash. D. C.). 169 : 1095.

2. Lehrer, R. I., and M. J. Cline. 1969. Leukocyte myeloperoxidase deficiency and disseminated candidiasis: the role of myeloperoxidase in resistance to Candida infection. J. Clin. Invest. 48 : 1478.

3. Lehrer, R. I., J. Hanifin, and M. J. Cline. 1969. Defective bactericidal activity in myeloperoxidase-deficient human neutrophils. Nature (Lond). 223: 78.

4. Klebanoff, S. J., and S. H. Pincus. 1971. Hydrogen peroxide utilization in myeloperoxidase-deficient leukocytes: a possible microbicidal control mechanism. $J$. Clin. Invest. 50: 2226.

5. Lehrer, R. I., and M. J. Cline. 1969. Interaction of Candida albicans with human leukocytes and serum. $J$. Bacteriol. 98 : 996.

6. Böyum, A. 1968. Isolation of mononuclear cells and granulocytes from human blood. Isolation of mononuclear cells by one centrifugation, and of granulocytes by combining centrifugation and sedimentation at $1 \mathrm{~g}$. Scand. J. Clin. Lab. Invest. Suppl. $97: 77$.

7. Lehrer, R. I. 1970. Measurement of candidacidal activity of specific leukocyte types in mixed cell populations. I. Normal, myeloperoxidase-deficient, and chronic granulomatous disease neutrophils. Infect. Immun. 2: 42.

8. Stauffer, J. F. 1957. Anaerobic measurements. In Manometric Techniques. By W. W. Umbreit, R. H. Burris,and J. F. Stauffer, Burgess Publishing Co., Minneapolis, Minn. 3rd edition. 69.

9. Pincus, S. H., and S. J. Klebanoff. 1971. Quantitative leukocyte iodination. $N$. Engl. J. Med. 284: 744.

10. Lehrer, R. I. 1969. Antifungal effects of peroxidase systems. J. Bacteriol. 99 : 361.

11. Lehrer, R. I. 1971. Inhibition by sulfonamides of the candidacidal activity of human neutrophils. J. Clin. Invest. 50 : 2498.

12. Klebanoff, S. J. 1967. Iodination of bacteria: a bactericidal mechanism. J. Exp. Med. 126: 1063.

13. McCall, C. E., L. R. DeChatelet, M. R. Cooper, and P. Ashburn. 1971. The effects of ascorbic acid on bactericidal mechanisms of neutrophils. J. Infect. Dis. 124: 194.

14. Klebanoff, S. J. 1970. Myeloperoxidase-mediated antimicrobial systems and their role in leukocyte function. In Biochemistry of the Phagocytic Process: Localization and the Role of Myeloperoxidase and the Mechanism of the Halogenation Reaction. J. Schultz, editor. North Holland Publishing Co., Amsterdam. 89.

15. Holmes, B., A. R. Page, and R. A. Good. 1967. Studies of the metabolic activity of leukocytes from patients with a genetic abnormality of phagocytic function. $J$. Clin. Invest. 46: 1422. 